\title{
Breaking strong symmetries in dissipative quantum systems: Bosonic atoms coupled to a cavity
}

\author{
Catalin-Mihai Halati, Ameneh Sheikhan, and Corinna Kollath \\ Physikalisches Institut, University of Bonn, Nussallee 12, 53115 Bonn, Germany
}

(Received 25 January 2021; accepted 11 January 2022; published 11 February 2022)

\begin{abstract}
In dissipative quantum systems, strong symmetries can lead to the existence of conservation laws and multiple steady states. In this Letter we investigate a strong symmetry for bosonic atoms coupled to an optical cavity, an experimentally relevant system, generalizing the adiabatic elimination techniques and using numerically exact matrix product state methods. We show that for ideal bosons coupled to the cavity multiple steady states exist and in each symmetry sector a dissipative phase transition occurs at a different critical point. This implies that phases of very different natures can coexist. We find that the introduction of a slight breaking of the strong symmetry by a small interaction term leads to a direct transition from multiple steady states to a unique steady state. We point out the phenomenon of dissipative freezing, the breaking of the conservation law at the level of individual realizations in the presence of strong symmetry. For a small breaking of the strong symmetry we see that the behavior of the individual trajectories still shows some signs of this dissipative freezing before it fades out for larger symmetry breaking terms.
\end{abstract}

DOI: 10.1103/PhysRevResearch.4.L012015

Symmetries play a key role in classifying and unifying the physics occurring in different microscopic systems. A famous example is the universal behavior arising at (quantum) phase transitions. This universal behavior is independent of the microscopic details of the system and can be classified by the symmetries which are spontaneously broken at the transition. Typically, each symmetry of a Hamiltonian is connected to a conservation law. This has the crucial consequence that the long time state remembers the initial conditions and that the conservation laws need to be considered constructing thermal ensembles [1], the so-called generalized Gibbs ensembles [2].

Surprisingly, in contrast to the Hamiltonian case, for systems described by the dissipative Lindblad master equations $\frac{\partial}{\partial t} \rho=\mathcal{L}(\rho)$, where $\rho$ is the density matrix and $\mathcal{L}$ the Liouvillian, a symmetry of the Liouvillian does not always imply a conserved quantity and multiple steady states $[3,4]$. Let the Hermitian operator $\mathcal{O}$ be the generator of the symmetry $\mathcal{U}=\exp (i \phi \mathcal{O})$, with real $\phi$. If the symmetry operator satisfies the condition $\mathcal{L}\left(\mathcal{U} \rho \mathcal{U}^{\dagger}\right)=\mathcal{U} \mathcal{L}(\rho) \mathcal{U}^{\dagger}$, we have only a so-called weak symmetry. This weak symmetry condition is not sufficient to imply the existence of a conserved quantity or multiple steady states. Only if additionally $\mathcal{O}$ is commuting with both the Hamiltonian and all jump operators $J_{m},[\mathcal{O}, H]=\left[\mathcal{O}, J_{m}\right]=0$, a so-called strong symmetry exists which implies conservation of $\langle\mathcal{O}\rangle=\operatorname{tr}(\rho O)$ and multiple steady states.

Published by the American Physical Society under the terms of the Creative Commons Attribution 4.0 International license. Further distribution of this work must maintain attribution to the author(s) and the published article's title, journal citation, and DOI.
Recently, the consequences of weak and strong symmetries in open systems were discussed in the context of error correction for quantum information theory [5].

Experimental systems that can be described by a Lindblad master equation are very frequent in the area of quantum optics and solid state systems coupled to light. In many situations, a unique steady state arises. However, in recent years a significant amount of work has been devoted to go beyond this typical situation and to study the coexistence of several states in such Lindblad systems [6-18] and the phenomena of bi-/metastability [15,19-28] or intermittency [13-15,25,29]. Additionally, steady states with sought-after properties have been constructed employing symmetries of the system, such as steady states with $\eta$-pairing correlations [30] or states with enhanced currents [31,32] or in weakly driven systems [33].

In this Letter we show for a realistic experimental system, a cavity coupled to a quantum gas, how the presence of strong symmetry can lead to the occurrence of multiple dissipative phase transitions in different symmetry sectors. We identify that the phase transition can occur for different critical values depending on the considered symmetry sector. Thus, for the same physical parameters the nature of the steady state can be very different depending on the initial state of the system.

We further investigate how in the situation of the slight breaking of strong symmetry by an additional term in the Liouvillian a unique steady state is recovered. Thus, slight breaking causes a drastic response of the system. We investigate the timescales associated with the process of reaching the unique steady state. Additionally, we show the absence of intermittency, dissipative freezing [34], in the presence of strong symmetry in single trajectories obtained by the stochastic unraveling of the master equation. We find that this behavior of the absence of intermittency can approximately survive for an intermediate time when adding a small symmetry breaking term, whereas for a larger symmetry breaking term the 
different symmetry sectors are no longer a good description of the system.

We consider ultracold bosons confined to a onedimensional chain coupled to a single cavity mode and transversely pumped with a standing-wave laser beam [35]. Adiabatically eliminating the excited internal state of the atoms, the dynamics follows the Lindblad equation [35-39]

$\frac{\partial}{\partial t} \rho=\mathcal{L}(\rho)=-\frac{i}{\hbar}[H, \rho]+\frac{\Gamma}{2}\left(2 a \rho a^{\dagger}-a^{\dagger} a \rho-\rho a^{\dagger} a\right)$,

where $\mathcal{L}(\rho)$ is the Liouvillian. The bosonic operators $a$ and $a^{\dagger}$ are the annihilation and creation operators for the photon mode of the cavity. The dissipator with strength $\Gamma$ represents the losses from the cavity due to the imperfections of the mirrors. The Hamiltonian $H=H_{0}+H_{\text {int }}$ is given by [38-40]

$$
\begin{aligned}
H_{0}= & \hbar \delta a^{\dagger} a-\hbar \Omega\left(a+a^{\dagger}\right) \sum_{j} b_{k_{j}}^{\dagger} b_{k_{j}+\pi(\bmod 2 \pi)} \\
& -2 J \sum_{j} \cos \left(k_{j}\right) b_{k_{j}}^{\dagger} b_{k_{j}}, \\
H_{\text {int }}= & \frac{U}{2} \sum_{l} n_{l}\left(n_{l}-1\right) .
\end{aligned}
$$

The cavity mode is described by the first term in $H_{0}$, in the rotating frame of the pump beam, where $\delta=\omega_{c}-\omega_{p}$ is the detuning between the cavity mode and the transverse pump beam. The operators $b_{k_{j}}$ and $b_{k_{j}}^{\dagger}$ are the bosonic annihilation and creation operators of the atoms with the unitless momentum $k_{j}=\frac{2 \pi j}{L}$ and $j=1, \ldots, L$, assuming periodic boundary conditions. In the numerical results we considered open boundary conditions [41]. $J$ is the tunneling amplitude of the atoms and $U \geqslant 0$ the strength of the on-site interaction, where $l$ denotes the site of the chain and $n_{l}$ the atomic density. $L$ denotes the number of sites of the bosonic chain and the total number of bosons is $N$. The coupling between the atoms and the cavity field introduces a change of the momentum $k_{j}$ and $k_{j}+\pi(\bmod 2 \pi)$. This is due to the periodicity of the cavity mode which has twice the periodicity of the lattice spacing within the chain. The commensurability of the chain and the cavity field is an important condition for the realization of strong symmetry.

For $U=0$, only transitions between the occupation of the momenta $k_{j}$ and $k_{j}+\pi(\bmod 2 \pi)$ of the atoms are possible. In the single-particle case, $L / 2$ independent symmetry sectors exist, each spanned by the momentum states $\left|k_{j}\right\rangle$ and $\left|k_{j}+\pi(\bmod 2 \pi)\right\rangle, j=1, \ldots, L / 2$. These correspond to the strong symmetry, having as a generator the atomic number operators in each symmetry sector, $\mathcal{O}_{k_{j}}=b_{k_{j}}^{\dagger} b_{k_{j}}+$ $b_{k_{j}+\pi(\bmod 2 \pi)}^{\dagger} b_{k_{j}+\pi(\bmod 2 \pi)}$, and their average values are conserved quantities, $m_{k_{j}}=\left\langle\mathcal{O}_{k_{j}}\right\rangle$. Due to the strong symmetry, multiple steady states already exist for a single particle.

For $N$ atoms, the symmetry sectors can be constructed from the different combinations in which one can arrange the atoms in the single-particle sectors. Thus, each symmetry sector will be labeled by $K \equiv\left(m_{k_{1}}, \ldots, m_{k_{i}}, \ldots, m_{k_{L / 2}}\right)$, with $\sum_{i=1}^{L / 2} m_{k_{i}}=N$. However, even though the atoms can be

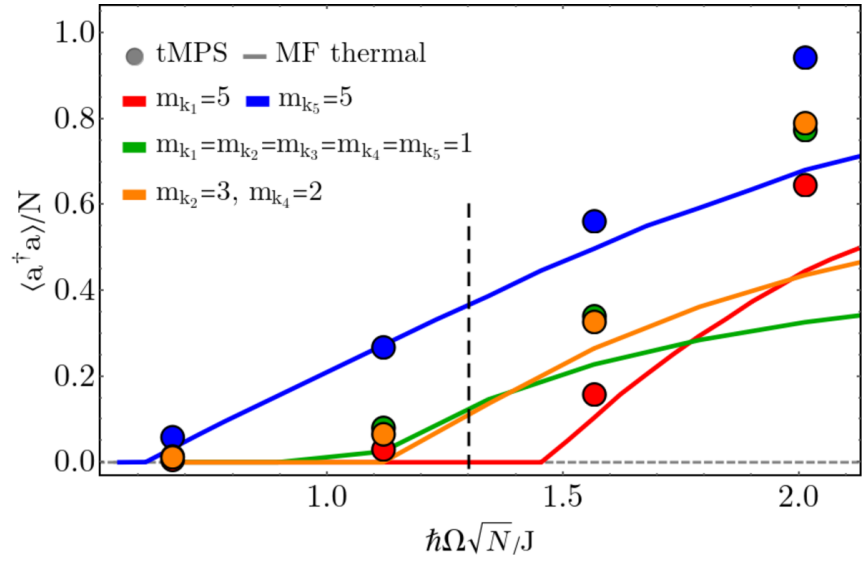

FIG. 1. The scaled photon number $\left\langle a^{\dagger} a\right\rangle / N$, as a function of $\hbar \Omega \sqrt{N} / J$, for different symmetry sectors. We compare the values obtained with time-dependent matrix product state (tMPS) at $t \mathrm{~J} / \hbar=$ 200 with the generalized Gibbs ensemble within the mean-field approach including thermal fluctuations. The parameters used are $L=10, N=5, \hbar \delta / J=2, U / J=0$, and $\hbar \Gamma / J=1$. At the dashed vertical line, the nature of the obtained steady states reaches from states with an empty cavity (red symbol/line) to the self-organized states (remaining ones).

arranged independently in the single-particle sectors, they are coupled via the photon field.

Nature of the steady states. We show in Fig. 1 the steady state diagram of a coupled atom cavity system. We observe in each considered symmetry sector of the strong symmetry a transition from the empty cavity state to the self-organized state with finite cavity occupation. Importantly, the transitions in the different symmetry sectors take place at distinct critical values of the coupling strength $\Omega_{c}$. Physically, this can be understood in the following way: The self-organization transition arises due to a competition between the ordering of the atoms in a density wave induced by an interaction with the photon mode and the kinetic energy of the atoms which depends on the momentum of the atoms. As in each symmetry sector the atoms have different momenta, this gives rise to different critical values for the transition. Thus, at a fixed coupling strength (cf. vertical line in Fig. 1) multiple steady states arise depending on the projection of the initial state to the symmetry sectors. For the considered strong symmetry, these steady states can even have very different natures. It can occur that one sector is still in the disordered phase with an empty cavity, whereas another sector already is deep in the self-organized phase. This is to be contrasted to the metastable states arising for weak symmetries, which are typically connected to a unique steady state.

The results presented in Fig. 1 are obtained using two different methods. The first approach is a mean-field decoupling of the atomic and the photonic sector considering the fluctuations around the mean-field solution as a perturbation together with the assumption that the atoms thermalize $[41,42]$. In the presence of strong symmetry we generalize this many-body adiabatic elimination approach by considering the conservation laws and using the thermalization of the atoms within each $[k, k+\pi(\bmod 2 \pi)]$ sector with sector-dependent temperatures. This corresponds to a generalization of the 
"generalized" Gibbs ensemble to dissipative systems [41]. We verify its applicability with an independent method. We expect that this generalization of the methods to be widely used in the future to consider dissipative systems in the presence of symmetries. Our second approach is a matrix product state (MPS) method developed [43] for the numerically exact simulation of the time evolution of the dissipative master equation, Eqs. (1) and (2) (the numerical parameters are given in the Supplemental Material [41]). For the time evolution we have chosen the empty cavity and the ground state in the atomic sector as the initial state.

Dissipative freezing. Recently, the effect of the existence of strong symmetry in a Liouvillian on the time evolution of the quantum trajectories was analyzed and the phenomenon of "dissipative freezing" was shown for systems in which the Lindblad operator is proportional to the Hamiltonian $(H \propto L)$ [34]. Dissipative freezing is the phenomenon where single realizations of trajectories, obtained by the stochastic unraveling of the master equation, can break the strong symmetry. A trajectory which is purely in one symmetry sector will remain in that sector for the rest of the time evolution and thus obey the symmetry of the system. However, starting with an initial state which is a superposition with contributions from multiple symmetry sectors, each individual trajectory will randomly select one of the sectors and remain there for the rest of the evolution. Further, no intermittency occurs between these trajectories in different sectors. Thus, even though the Monte Carlo average expectation value of the generator of the symmetry is a conserved quantity, this is no longer true at the level of single trajectories. The single trajectories can break "spontaneously" the strong symmetry of the model. This is an intriguing effect which might have relevance to the single realizations of experiments. Further, the interpretation of quantities measured in the quantum trajectory method which stabilize at different values, should not imply different steady states, but reflect the initial superposition.

Here, we numerically show [Figs. 2(a) and 2(b)] that even in a system that goes beyond the special case $(H \propto L)$ of Ref. [34], dissipative freezing can occur. We show the evolution of the initial state which is an equal superposition of a state from the sector $\left(m_{k_{1}}=5\right)$ and the sector $\left(m_{k_{1}}=1, m_{k_{2}}=\right.$ $\left.1, m_{k_{3}}=1, m_{k_{4}}=1, m_{k_{5}}=1\right)$. We can observe that at long times, $t J \gtrsim 40 \hbar$, all trajectories evolved to one of the two symmetry sectors, as $\left\langle\mathcal{O}_{k}\right\rangle(t)$ equals the expected occupation in those sectors. The Monte Carlo average of the trajectories stays constant throughout the following time evolution, up to a numerical error. Figures 2(c)-2(f) correspond to a finite on-site interaction and will be discussed later.

Breaking of the strong symmetry. For any finite interaction, $U>0$, the operators $\mathcal{O}_{k}$ no longer commute with the Hamiltonian, thus the strong symmetry of the Liouvillian is broken. We analyze how the system passes over from having multiple steady states to a unique steady state as the on-site interaction is slowly turned on. We focus on the limit of large dissipation, for which the generalized many-body adiabatic elimination predicts the steady state transition between the multiple steady states at $U=0, \rho_{K, \text { st }}[41]$ to a single steady state which is the totally mixed state $\rho_{\text {mix }}[41,43]$.

In Figs. 3(a) and 3(b) the behavior of the expectation value of the photon number and the conserved quantities of the
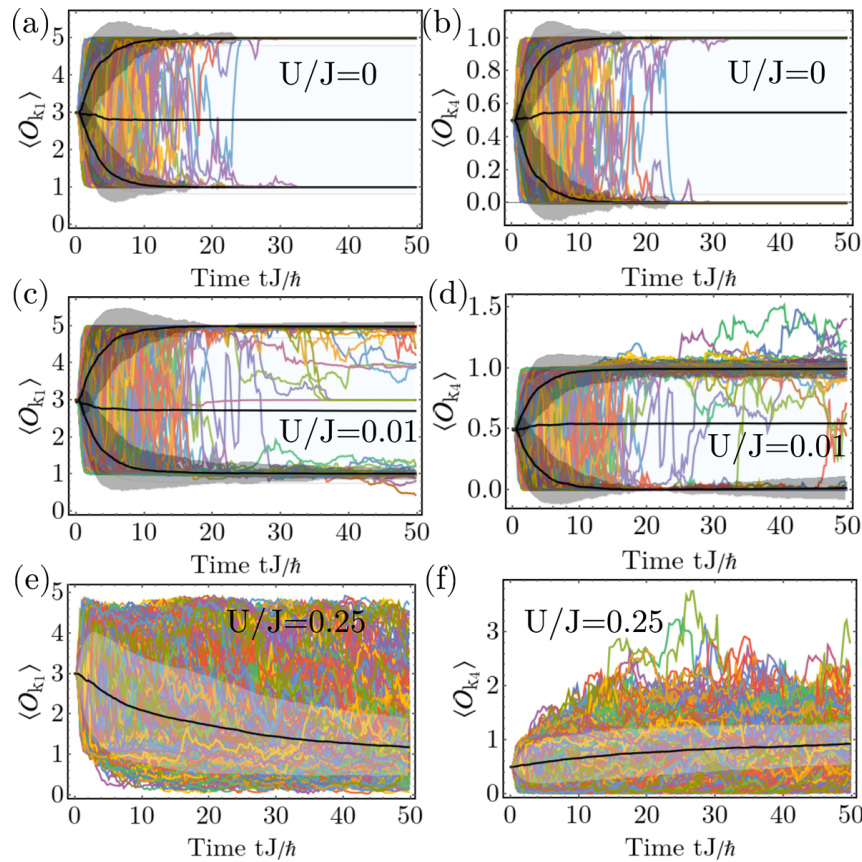

FIG. 2. Time evolution of $\mathcal{O}_{k}$ for the single quantum trajectories sampled in the Monte Carlo average for different interaction strengths $U$, with $k=k_{1}$ (left column) and $k=k_{4}$ (right column). The initial state consists in an equal superposition between states for the sectors $\left(m_{k_{1}}=5\right)$ and $\left(m_{k_{1}}=1, m_{k_{2}}=1, m_{k_{3}}=1, m_{k_{4}}=\right.$ $\left.1, m_{k_{5}}=1\right)$. In each panel there are 1000 trajectories plotted, where the black represents the Monte Carlo average, either for the full set of trajectories, or averaged separately depending on the final value, and we shade the interval of one standard deviation away from the average, with light blue for the full average and light gray for the separate averages. The parameters used are $L=10, N=5$, $\hbar \delta / J=2, \hbar \Omega \sqrt{N} / J=4.47$, and $\hbar \Gamma / J=15$.

symmetry at fixed time $t J=49.75 \hbar$ is plotted as a function of the interaction strength. We consider initial states in different symmetry sectors. For these, we observe that at $U=0$ multiple steady states are obtained as signaled by distinct expectation values. However, as the interaction strength is increased the values of the photon number and $\left\langle\mathcal{O}_{k}\right\rangle$ for the different initial states start to be more and more similar until they agree with each other and with the values expected for $\rho_{\text {mix }}$, for large values of interaction $U$. The deviations from the predicted unique steady state $\rho_{\text {mix }}$ for small interaction strengths are due to the fact that the system has not yet reached its steady state at the shown time. This can be observed in time-evolution plots given in Figs. 3(c) and 3(d) for the photon number and $\left\langle\mathcal{O}_{k}\right\rangle-\left\langle\mathcal{O}_{k}\right\rangle_{\rho_{\text {mix }}}$ for different interaction values. The expected steady state value for $\left\langle a^{\dagger} a\right\rangle / N$ is represented with a gray line in Fig. 3(c) and $\left\langle\mathcal{O}_{k}\right\rangle_{\rho_{\text {mix }}}=1$. In order to quantify this we fitted the time dependence of $\left\langle a^{\dagger} a\right\rangle-\left\langle a^{\dagger} a\right\rangle_{\rho_{\text {mix }}}$ and $\left\langle\mathcal{O}_{k}\right\rangle-\left\langle\mathcal{O}_{k}\right\rangle_{\rho_{\operatorname{mix}}}$ with an exponential function $\propto e^{-t / \tau}$, and extracted the timescales for reaching the steady state. The fits describe very nicely the numerical data, which gives strong support that at infinite time the steady state is given by $\rho_{\text {mix }}$. Additionally, the dependence of the timescale on $U$ is represented in Figs. 3(e) and 3(f) in log-log plots. The timescales exhibit an algebraic dependence on $1 / U^{2}$. We compare this 

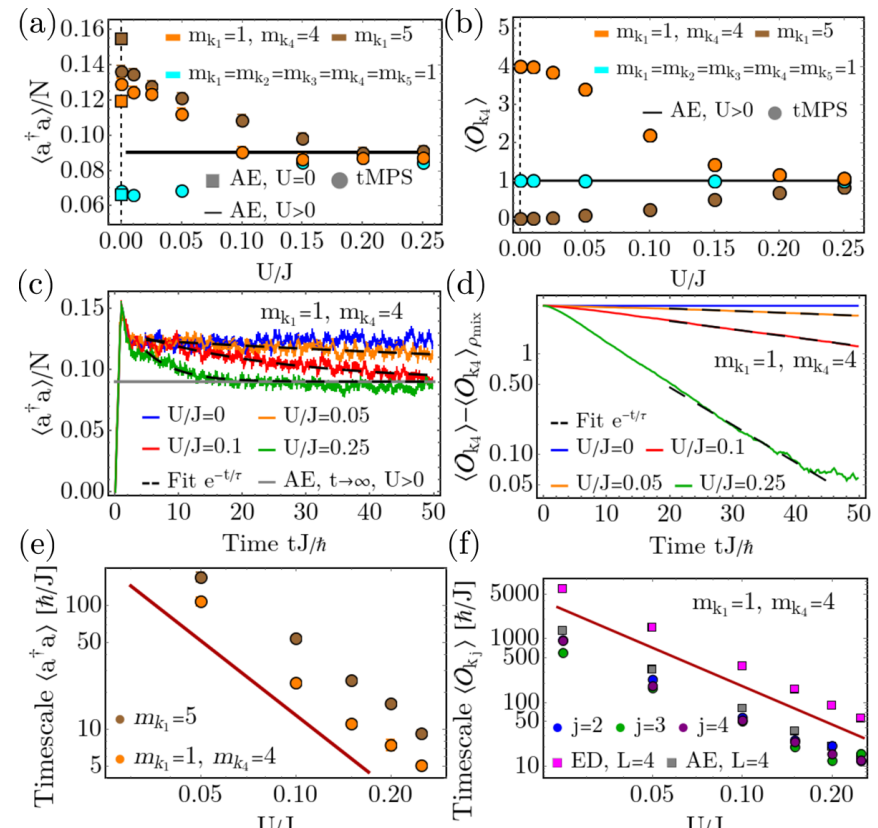

(f)

$$
\text { Time } \mathrm{t} J / \hbar
$$

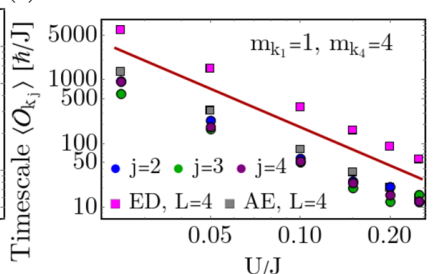

FIG. 3. The dependence on the interaction strength $U$ of (a) the scaled photon number $\left\langle a^{\dagger} a\right\rangle / N$ and (b) the expectation value of $\mathcal{O}_{k_{4}}$ using tMPS at time $t J=49.75 \hbar$ and many-body adiabatic elimination (AE). The time evolution of (c) the scaled photon number $\left\langle a^{\dagger} a\right\rangle / N$ and (d) the expectation value of $\mathcal{O}_{k_{4}}$ for different values of $U$. For finite $U$ we fit the time evolution with an exponential decay (black dashed lines), the difference between the tMPS data and the expected steady state value, obtained from many-body adiabatic elimination, using the kinetic energy as a perturbation. The timescales obtained from the exponential fits are plotted as a function of $U$ in log-log scale for (e) $\left\langle a^{\dagger} a\right\rangle / N$ and (f) $\mathcal{O}_{k_{j}}$. In (f) we compare the timescales of $\mathcal{O}_{k_{j}}$ with the longest timescale of a small system of $N=2$ particles in an $L=4$ system computed with exact diagonalization (ED) and many-body adiabatic elimination (AE). We fit the timescale dependence on the interaction with an algebraic decay $\propto U^{-\alpha}$ and obtain the following exponents: (e) $\left(m_{k_{1}}=5\right)$, $\alpha=1.86 \pm 0.07$; $\left(m_{k_{1}}=1, m_{k_{4}}=4\right), \alpha=1.73 \pm 0.08$; (f) $j=2$, $\alpha=1.92 \pm 0.06 ; j=3, \alpha=1.77 \pm 0.09 ; j=4, \alpha=1.78 \pm 0.03$. The red lines are a guide to the eye of an algebraic decay $\propto U^{-2}$. The parameters are chosen to be $L=10, N=5, \hbar \Omega \sqrt{N} / J=4.47$, $\hbar \delta / J=2$, and $\Gamma / J=15$.

behavior with results from the exact diagonalization of either the full Liouvillian Eq. (1) (ED) or the many-body adiabatic elimination equations of motion, with the kinetic energy as a perturbation [43] (AE), for a small system of $L=4$. In these cases we compute the timescale as the inverse of the real part of the first excited eigenvalue and we obtain an algebraic dependence $\alpha U^{-\alpha}$ with $\alpha \approx 2$ confirming the tMPS results. We observe that the timescales for $\left\langle\mathcal{O}_{k}\right\rangle$ are consistently larger than the timescales for the photon number, which signals that in our simulations the photon state is reaching the steady state before the atomic one. We attribute this to the spatial extent of the atomic system.

Thus, we see that the time evolution at short times remembers well the strong symmetry and the mixing of the different symmetry sectors only occurs on timescales $\propto 1 / U^{2}$ associated with the scattering of the atoms.

A question which arises is how the breaking of the symmetry affects the phenomenon of dissipative freezing discussed before. We study in the following how this phenomenon is affected by the presence of a small interaction, the symmetry breaking term.

We observe in Figs. 2(c) and 2(d) that at $U / J=0.01$ the time evolution found for the single trajectories resembles at an early time the one at $U / J=0$. This means that the single trajectories break the approximate strong symmetry and approach the two different symmetry sectors. At an intermediate time $20 \lesssim t J / \hbar$ many of the quantum trajectories spend a long time near the two values expected from the $U=0$ symmetry sectors. Only few of the trajectories directly show deviations from these values or intermittency between the values such that the phenomenon of dissipative freezing also occurs here to an approximate extent. One has to be careful not to misinterpret this absence of intermittency as the existence of a multiple steady state. Only starting from $U / J \gtrsim 0.25$ the effect of the strong symmetry washes out in the considered time interval.

To summarize, we analyzed the effects of a strong symmetry and of a slight breaking of this symmetry on the dynamics of a many-body open system consisting of bosonic atoms coupled to an optical cavity. The strong symmetry stems from the commensurability of the one-dimensional atomic chain with the cavity field. Such a strong symmetry implies the existence of multiple steady states and we showed that the dissipative phase transition to the self-organized state can occur at different thresholds in different symmetry sectors described by generalized Gibbs ensembles in the atomic part. We analyzed how the nature of the steady state changes drastically when a small term that breaks the strong symmetry is introduced. The timescales towards the unique steady state with altered properties were found to be proportional to $1 / U^{2}$ associated with the scattering between the atoms. We have shown that even for a many-body system with strong symmetry the phenomenon of dissipative freezing can occur when one considers the behavior of individual quantum trajectories. It appears that, at an intermediate time, one can still identify the effect of dissipative freezing even if strong symmetry has been slightly broken. An open question remains whether spontaneous symmetry breaking can also be observed in single trajectories of an experimental measurement. This would question the interpretation of the absence of intermittency in experimental measurements.

Acknowledgments. We thank J.-S. Bernier, A. V. Bezvershenko, M. Fleischhauer, A. Rosch, and S. Wolff for stimulating discussions. We acknowledge funding from the Deutsche Forschungsgemeinschaft (DFG, German Research Foundation) in particular under Projects No. 277625399 TRR 185 (B3), No. 277146847 - CRC 1238 (C05), and No. KO 4771/4-1, FOR1807 (P10) and under Germany's Excellence Strategy - Cluster of Excellence Matter and Light for Quantum Computing (ML4Q) EXC 2004/1 - 390534769 and the European Research Council (ERC) under the Horizon 2020 research and innovation programme, Grant Agreement No. 648166 (Phonton). 
[1] R. Balian, From Microphysics to Macrophysics (Springer, Berlin, 2007).

[2] M. Rigol, V. Dunjko, V. Yurovsky, and M. Olshanii, Relaxation in a Completely Integrable Many-Body Quantum System: An $A b$ Initio Study of the Dynamics of the Highly Excited States of 1D Lattice Hard-Core Bosons, Phys. Rev. Lett. 98, 050405 (2007).

[3] B. Buča and T. Prosen, A note on symmetry reductions of the Lindblad equation: Transport in constrained open spin chains, New J. Phys. 14, 073007 (2012).

[4] V. V. Albert and L. Jiang, Symmetries and conserved quantities in Lindblad master equations, Phys. Rev. A 89, 022118 (2014).

[5] S. Lieu, R. Belyansky, J. T. Young, R. Lundgren, V. V. Albert, and A. V. Gorshkov, Symmetry Breaking and Error Correction in Open Quantum Systems, Phys. Rev. Lett. 125, 240405 (2020).

[6] E. M. Kessler, G. Giedke, A. Imamoglu, S. F. Yelin, M. D. Lukin, and J. I. Cirac, Dissipative phase transition in a central spin system, Phys. Rev. A 86, 012116 (2012).

[7] F. Minganti, A. Biella, N. Bartolo, and C. Ciuti, Spectral theory of Liouvillians for dissipative phase transitions, Phys. Rev. A 98, 042118 (2018).

[8] H. J. Carmichael, Breakdown of Photon Blockade: A Dissipative Quantum Phase Transition in Zero Dimensions, Phys. Rev. X 5, 031028 (2015).

[9] H. Weimer, Variational Principle for Steady States of Dissipative Quantum Many-Body Systems, Phys. Rev. Lett. 114, 040402 (2015).

[10] M. Benito, C. Sánchez Muñoz, and C. Navarrete-Benlloch, Degenerate parametric oscillation in quantum membrane optomechanics, Phys. Rev. A 93, 023846 (2016).

[11] L. M. Sieberer, S. D. Huber, E. Altman, and S. Diehl, Dynamical Critical Phenomena in Driven-Dissipative Systems, Phys. Rev. Lett. 110, 195301 (2013).

[12] C. Sánchez Muñoz, A. Lara, J. Puebla, and F. Nori, Hybrid Systems for the Generation of Nonclassical Mechanical States via Quadratic Interactions, Phys. Rev. Lett. 121, 123604 (2018).

[13] M. Biondi, G. Blatter, H. E. Türeci, and S. Schmidt, Nonequilibrium gas-liquid transition in the driven-dissipative photonic lattice, Phys. Rev. A 96, 043809 (2017).

[14] M.-J. Hwang, P. Rabl, and M. B. Plenio, Dissipative phase transition in the open quantum Rabi model, Phys. Rev. A 97, 013825 (2018).

[15] J. J. Mendoza-Arenas, S. R. Clark, S. Felicetti, G. Romero, E. Solano, D. G. Angelakis, and D. Jaksch, Beyond mean-field bistability in driven-dissipative lattices: Bunching-antibunching transition and quantum simulation, Phys. Rev. A 93, 023821 (2016).

[16] H. Wilming, M. J. Kastoryano, A. H. Werner, and J. Eisert, Emergence of spontaneous symmetry breaking in dissipative lattice systems, J. Math. Phys. 58, 033302 (2017).

[17] J. Hannukainen and J. Larson, Dissipation-driven quantum phase transitions and symmetry breaking, Phys. Rev. A 98, 042113 (2018).

[18] J. S. Ferreira and P. Ribeiro, Lipkin-Meshkov-Glick model with Markovian dissipation: A description of a collective spin on a metallic surface, Phys. Rev. B 100, 184422 (2019).

[19] K. Macieszczak, M. Guţă, I. Lesanovsky, and J. P. Garrahan, Towards a Theory of Metastability in Open Quantum Dynamics, Phys. Rev. Lett. 116, 240404 (2016).
[20] T. Fink, A. Schade, S. Höfling, C. Schneider, and A. Imamoglu, Signatures of a dissipative phase transition in photon correlation measurements, Nat. Phys. 14, 365 (2018).

[21] C. Carr, R. Ritter, C. G. Wade, C. S. Adams, and K. J. Weatherill, Nonequilibrium Phase Transition in a Dilute Rydberg Ensemble, Phys. Rev. Lett. 111, 113901 (2013).

[22] N. R. de Melo, C. G. Wade, N. Šibalić, J. M. Kondo, C. S. Adams, and K. J. Weatherill, Intrinsic optical bistability in a strongly driven Rydberg ensemble, Phys. Rev. A 93, 063863 (2016).

[23] S. R. K. Rodriguez, W. Casteels, F. Storme, N. Carlon Zambon, I. Sagnes, L. Le Gratiet, E. Galopin, A. Lemaître, A. Amo, C. Ciuti, and J. Bloch, Probing a Dissipative Phase Transition via Dynamical Optical Hysteresis, Phys. Rev. Lett. 118, 247402 (2017).

[24] F. Letscher, O. Thomas, T. Niederprüm, M. Fleischhauer, and H. Ott, Bistability Versus Metastability in Driven Dissipative Rydberg Gases, Phys. Rev. X 7, 021020 (2017).

[25] P. R. Muppalla, O. Gargiulo, S. I. Mirzaei, B. P. Venkatesh, M. L. Juan, L. Grünhaupt, I. M. Pop, and G. Kirchmair, Bistability in a mesoscopic Josephson junction array resonator, Phys. Rev. B 97, 024518 (2018).

[26] M. J. A. Schuetz, E. M. Kessler, L. M. K. Vandersypen, J. I. Cirac, and G. Giedke, Steady-State Entanglement in the Nuclear Spin Dynamics of a Double Quantum Dot, Phys. Rev. Lett. 111, 246802 (2013).

[27] M. J. A. Schuetz, E. M. Kessler, L. M. K. Vandersypen, J. I. Cirac, and G. Giedke, Nuclear spin dynamics in double quantum dots: Multistability, dynamical polarization, criticality, and entanglement, Phys. Rev. B 89, 195310 (2014).

[28] L. Hruby, N. Dogra, M. Landini, T. Donner, and T. Esslinger, Metastability and avalanche dynamics in strongly correlated gases with long-range interactions, Proc. Natl. Acad. Sci. U.S.A. 115, 3279 (2018).

[29] M. Fitzpatrick, N. M. Sundaresan, A. C. Y. Li, J. Koch, and A. A. Houck, Observation of a Dissipative Phase Transition in a One-Dimensional Circuit QED Lattice, Phys. Rev. X 7, 011016 (2017).

[30] J.-S. Bernier, P. Barmettler, D. Poletti, and C. Kollath, Emergence of spatially extended pair coherence through incoherent local environmental coupling, Phys. Rev. A 87, 063608 (2013).

[31] D. Manzano and P. I. Hurtado, Symmetry and the thermodynamics of currents in open quantum systems, Phys. Rev. B 90, 125138 (2014).

[32] F. Lange, Z. Lenarčič, and A. Rosch, Pumping approximately integrable systems, Nat. Commun. 8, 15767 (2017).

[33] Z. Lenarčič, F. Lange, and A. Rosch, Perturbative approach to weakly driven many-particle systems in the presence of approximate conservation laws, Phys. Rev. B 97, 024302 (2018).

[34] C. Sánchez Muñoz, B. Buča, J. Tindall, A. González-Tudela, D. Jaksch, and D. Porras, Symmetries and conservation laws in quantum trajectories: Dissipative freezing, Phys. Rev. A 100, 042113 (2019).

[35] C.-M. Halati, A. Sheikhan, H. Ritsch, and C. Kollath, Numerically Exact Treatment of Many-Body Self-Organization in a Cavity, Phys. Rev. Lett. 125, 093604 (2020).

[36] H. Carmichael, An Open Systems Approach to Quantum Optics (Springer, Berlin, 1991).

[37] H. P. Breuer and F. Petruccione, The Theory of Open Quantum Systems (Oxford University Press, Oxford, UK, 2002). 
[38] H. Ritsch, P. Domokos, F. Brennecke, and T. Esslinger, Cold atoms in cavity-generated dynamical optical potentials, Rev. Mod. Phys. 85, 553 (2013).

[39] C. Maschler, I. B. Mekhov, and H. Ritsch, Ultracold atoms in optical lattices generated by quantized light fields, Eur. Phys. J. D 46, 545 (2008).

[40] G. S. D. Nagy and P. Domokos, Self-organization of a BoseEinstein condensate in an optical cavity, Eur. Phys. J. D 48, 127 (2008).

[41] See Supplemental Material at http://link.aps.org/supplemental/ 10.1103/PhysRevResearch.4.L012015 for derivations of the generalized many-body adiabatic elimination equations of motion and steady states, additional comparisons with the numerical tMPS results, and details on the time-dependent matrix product state approach (tMPS), which includes Refs. [33-35,42-57].

[42] A. V. Bezvershenko, C.-M. Halati, A. Sheikhan, C. Kollath, and A. Rosch, Dicke Transition in Open Many-Body Systems Determined by Fluctuation Effects, Phys. Rev. Lett. 127, 173606 (2021).

[43] C.-M. Halati, A. Sheikhan, and C. Kollath, Theoretical methods to treat a single dissipative bosonic mode coupled globally to an interacting many-body system, Phys. Rev. Research 2, 043255 (2020).

[44] J. J. García-Ripoll, S. Dürr, N. Syassen, D. M. Bauer, M. Lettner, G. Rempe, and J. I. Cirac, Dissipation-induced hardcore boson gas in an optical lattice, New J. Phys. 11, 013053 (2009).

[45] F. Reiter and A. S. Sørensen, Effective operator formalism for open quantum systems, Phys. Rev. A 85, 032111 (2012).

[46] D. Poletti, P. Barmettler, A. Georges, and C. Kollath, Emergence of Glasslike Dynamics for Dissipative and Strongly Interacting Bosons, Phys. Rev. Lett. 111, 195301 (2013).
[47] B. Sciolla, D. Poletti, and C. Kollath, Two-Time Correlations Probing the Dynamics of Dissipative Many-Body Quantum Systems: Aging and Fast Relaxation, Phys. Rev. Lett. 114, 170401 (2015).

[48] A. C. Y. Li, F. Petruccione, and J. Koch, Perturbative approach to Markovian open quantum systems, Sci. Rep. 4, 4887 (2014).

[49] C.-M. Halati, External control of many-body quantum systems, Ph.D. thesis, University of Bonn, 2021, https://bonndoc.ulb.unibonn.de/xmlui/handle/20.500.11811/9343.

[50] J. Dalibard, Y. Castin, and K. Mølmer, Wave-Function Approach to Dissipative Processes in Quantum Optics, Phys. Rev. Lett. 68, 580 (1992).

[51] C. W. Gardiner, A. S. Parkins, and P. Zoller, Wave-function quantum stochastic differential equations and quantum-jump simulation methods, Phys. Rev. A 46, 4363 (1992).

[52] A. J. Daley, Quantum trajectories and open many-body quantum systems, Adv. Phys. 63, 77 (2014).

[53] S. R. White and A. E. Feiguin, Real-Time Evolution Using the Density Matrix Renormalization Group, Phys. Rev. Lett. 93, 076401 (2004).

[54] A. J. Daley, C. Kollath, U. Schollwöck, and G. Vidal, Timedependent density-matrix renormalization-group using adaptive effective Hilbert spaces, J. Stat. Mech.: Theor. Exp. (2004) P04005.

[55] U. Schollwöck, The density-matrix renormalization group in the age of matrix product states, Ann. Phys. 326, 96 (2011).

[56] E. M. Stoudenmire and S. R. White, Minimally entangled typical thermal state algorithms, New J. Phys. 12, 055026 (2010).

[57] M. L. Wall, A. Safavi-Naini, and A. M. Rey, Simulating generic spin-boson models with matrix product states, Phys. Rev. A 94, 053637 (2016). 